\title{
Conservation Laws and Integrability Conditions for Gravitational and Yang-Mills Field Equations
}

\author{
Michel Dubois-Violette ${ }^{1}$ and John Madore ${ }^{2}$ \\ ${ }^{1}$ Laboratoire de Physique Théorique et Hautes Energies*, Université de Paris-Sud, Bâtiment 211, \\ F-91405 Orsay, France \\ ${ }^{2}$ Centre de Physique Théorique, Ecole Polytechnique, F-91128 Palaiseau, France
}

\begin{abstract}
We show that expressed in the appropriate principal bundles, the gravitational and Yang-Mills fields equations are equivalent to integrability conditions and that the latter can also be interpreted, using local sections, as conservation equations of the local pseudo-densities of energy-momentum and Yang-Mills charge respectively. These densities can be related using a KaluzaKlein unification.
\end{abstract}

\section{Introduction}

There is no satisfactory definition of local density of energy-momentum for the gravitational field. Since the gravitational field equations are of second order, reasonable expressions for energy-momentum density at a point must involve only the values of the field and its first derivative at that point. The equivalence principle implies that by a change of frame one can set these to zero. It follows then that expressions which are used to describe the local energy-momentum density cannot be tensorial. They are called "pseudo-tensorial" and are not uniquely defined. This property of energy-momentum density and the origin of the difficulty has led most physicists to the conclusion that the problem is ill-defined and the object irrelevant; density of gravitational energy is like "density of beauty of a painting." However, for asymptotically flat space-times, the total energy-momentum is a well-defined and useful concept as shown, for instance, by the fact that the total energy must be greater or equal to zero and vanish only for flat space [1-6].

Since to speak of local energy-momentum one must first fix a frame and since we know that any definition will be strongly frame dependent, it is natural to wonder whether on the frame bundle there is not a canonical expression such that the different energy-momentum densities on space-time, that is, the different pseudo-tensors, are obtained by pull-back in the different local sections of the frame bundle. On the general frame bundle, this is not quite true but we shall show that if we restrict our attention to the bundle of orthonormal frames, there is indeed such a canonical

\footnotetext{
* Laboratoire Associé au C.N.R.S.
} 
object which is a generalization of an expression given by Sparling [7] in the Lorentzian, 4-dimensional case.

Given an $n$-dimensional pseudo-Riemannian manifold $M$ with pseudo-metric $g$ of arbitrary signature, there exists an $\mathbb{R}^{n}$-valued $(n-1)$-form $\tau_{i}$ on the bundle $O(M, g)$ of orthonormal frames, constructed purely algebraically in terms of the canonical form $\theta^{i}$ and an arbitrary connection form $\omega_{j}^{i}$ on $O(M, g)$, with the property that the equation $d \tau_{i}=0$ is equivalent to the vanishing of the torsion of $\omega^{i}{ }_{j}\left(\omega_{j}^{i}\right.$ is the LeviCivita connection), and the vanishing of the Ricci tensor. It turns out that $\tau_{i}$ is exact whenever it is closed and that there is an $\mathbb{R}^{n}$-valued $(n-2)$-form $\sigma_{i}$ on $O(M, g)$, again constructed in a purely algebraic way from $\theta^{i}$ and $\omega_{j}^{i}$ such that $\mathrm{d} \tau_{i}=0$ is also equivalent to $\tau_{i}=\mathrm{d} \sigma_{i}$.

We also show that these results can be generalized to include sources. One can prescribe an arbitrary Ricci tensor provided that the appropriate compatibility condition with the Bianchi identity is satisfied. Explicitly, if $t_{i}$ is the lift to $O(M, g)$ of a vectorial $(n-1)$-form on $M$ satisfying the equation $d t_{i}+\omega_{i}{ }^{j} \wedge t_{j}=0$, where $\omega_{j}^{i}$ is again an arbitrary connection form on $O(M, g)$ and if $\tau_{i}$ and $\sigma_{i}$ are as above then the equation $d\left(\tau_{i}+t_{i}\right)=0$ is equivalent to the vanishing of the torsion of $\omega^{i}{ }_{j}$ and to the Einstein equations with sources. Furthermore $d\left(\tau_{i}+t_{i}\right)=0$ is also equivalent to $\tau_{i}+t_{i}=\mathrm{d} \sigma_{i}$.

Apart from their mathematical interest, these results yield the physical interpretation of $\tau_{i}$ and of $\sigma_{i}$ in $n$-dimensional General Relativity. The inverse images on $M$ of $\tau_{i}$ and $\sigma_{i}$ with respect to local sections give an expression for the local energy-momentum density of the gravitational field and for the corresponding potential. The inverse image of the form $t_{i}$ is to be obviously identified with the energy-momentum density of the sources. It is worth noting that if $\omega_{j}^{i}$ is the LeviCivita connection, the inverse image on $M$ by a section of $O(M, g)$ of the equation $\tau_{i}+t_{i}=d \sigma_{i}$ has been already identified with the Einstein equation (See [8], Sect. 4.2.11(b)). Notice also that since $\tau_{i}$ and $\sigma_{i}$ have vertical components, their inverse images are strongly section-dependent.

There is another situation where there is a similar problem in the definition of a local physical quantity, the charge density of a Yang-Mills field. Here the origin of the difficulty is gauge invariance. Since to produce "pseudo-densities" of charge, one must first fix the gauge, it is again natural to look for a canonical object on the appropriate principal bundle such that different pseudo-densities of charge on space-time can be obtained by pull back in different local sections, that is, in different local gauges. We show that this object exists and is analogous to the gravitational one. The two can be related using a Kaluza-Klein unification of gravity and YangMills fields.

In Sect. 1 we establish our notation and briefly recall the frame-bundle formalism. In Sect. 2 we generalize the original form introduced by Sparling to an $n$ dimensional manifold. In Sect. 3 the results of Sect. 2 are in turn generalized to include the form which is appropriate when considering the most general secondorder lagrangian field equations. In Sect. 4 the particularly interesting case of dimension 4 is considered. In Sect. 5 we discuss in a similar way Yang-Mills theory and relate the results to the previous ones using a Kaluza-Klein unification. We show that the decomposition along the base-space and internal-space components 
yields the classical pseudo-tensor and the corresponding pseudo-current from Yang-Mills theory.

\section{Notation}

Consider an $n$-dimensional, smooth, pseudo-Riemannian manifold $M$ with metric $g$ of arbitrary signature. Let $T(M)$ be the tangent bundle and $O(M, g)$ the principal bundle of orthonormal frames over $M$. The structure group of $O(M, g)$ is the subgroup $O(n-m, m)$ of $G L(n, \mathbb{R})$ which leaves the metric invariant. Let $\pi$ be the projection of $O(M, g)$ onto $M$. Let $X$ be a tangent vector at a point $x$ in $O(M, g)$. The canonical or soldering form $\theta$ is an $\mathbb{R}^{n}$-valued 1 -form on $O(M, g)$ whose $k^{\text {th }}$ component $\theta^{k}$ at $x$ on $X$ is the $k^{\text {th }}$ component of $\pi_{*}(X)$ in the frame $x$.

The connection form $\omega=\left(\omega_{j}^{i}\right)$ is a 1 -form on $O(M, g)$ which takes its values in the Lie algebra $o(n-m, m)$ of $O(n-m, m)$ and which satisfies the structure equations

$$
d \theta^{i}+\omega^{i}{ }_{j} \wedge \theta^{j}=\Theta^{j}, \quad d \omega^{i}{ }_{j}+\omega^{i}{ }_{k} \wedge \omega^{k}{ }_{j}=\Omega^{i}{ }_{j} .
$$

The $\mathbb{R}^{n}$-valued 2-form $\Theta^{i}$ is the torsion form and the $o(m-m, m)$-valued 2-form $\Omega^{i}{ }_{j}$ is the curvature form. By definition the connection form $\omega^{i}{ }_{j}$ is vertical and the canonical form $\theta^{i}$ is horizontal. The forms $\left(\omega_{j}^{i}, \theta^{i}\right)$ define an absolute parallelism in $O(M, g)$.

The torsion and the curvature satisfy the Bianchi identities

$$
\begin{gathered}
d \Theta^{i}+\omega^{i}{ }_{j} \wedge \Theta^{j}=\Omega^{i}{ }_{j} \wedge \theta^{j}, \\
d \Omega^{i}{ }_{j}+\omega^{i}{ }_{k} \wedge \Omega^{k}{ }_{j}-\Omega^{i}{ }_{k} \wedge \omega^{k}{ }_{j}=0 .
\end{gathered}
$$

The components of a type $(r, s)$ tensor field $T$ on $M$ in an orthonormal frame will be denoted $T^{i_{1} \cdots i_{r}}{ }_{j_{1} \cdots j_{s}}$ and indices will be raised and lowered using the canonical metric $g=\operatorname{diag}(-1, \ldots,-1,+1, \ldots,+1)$ with $n-m$ time-like, negative and $m$-space-like, positive eigenvalues.

The Riemann tensor is defined by the equation $\Omega^{i}{ }_{j}=1 / 2 R_{j k l}^{i} \theta^{k} \wedge \theta^{l}$, the Ricci tensor is given by $R_{i j}=R^{k}{ }_{i k j}$, and the Ricci scalar is $R=R_{i}^{i}$. For each integer $q$ with $0 \leqq q \leqq n$ we define the $(n-q)$-form $\theta^{*}{ }_{i_{1} \cdots i_{q}}$ by the equation

$$
\theta_{i_{1} \cdots i_{q}}=1 /(n-q) ! \varepsilon_{i_{1} \cdots i_{q}, i_{q+1} \cdots i_{n}} \theta^{l_{q+1}} \wedge \cdots \wedge \theta^{i_{n}}
$$

\section{The Generalized Sparling Form}

Define the $\mathbb{R}^{n}$-valued $(n-2)$-form $\sigma_{i}$ and $(n-1)$-form $\tau_{i}$ on $O(M, g)$ by the formulae

$$
\begin{aligned}
\sigma_{i} & =-1 / 2 \omega^{j k} \wedge \theta^{*}{ }_{i j k}, \\
\tau_{i} & =1 / 2\left(\omega_{i}{ }^{j} \wedge \omega^{k l} \wedge \theta^{*}{ }_{j k l}-\omega^{j}{ }_{l} \wedge \omega^{l k} \wedge \theta^{*}{ }_{i j k}\right) .
\end{aligned}
$$

It is easy to verify that they satisfy the following identities

$$
\begin{aligned}
d \sigma_{i}= & \tau_{i}+1 / 2\left(\Theta^{j} \wedge \omega^{k l} \wedge \theta^{*}{ }_{i j k l}-\Omega^{j k} \wedge \theta^{*}{ }_{i j k}\right), \\
d \tau_{i}= & \Theta^{m} \wedge 1 / 2\left(\omega_{i}{ }^{l} \wedge \omega^{j k} \wedge \theta^{*}{ }_{j k l m}-\omega^{j}{ }_{l} \wedge \omega^{l k} \wedge \theta^{*}{ }_{i j k m}\right) \\
& +1 / 2\left(\omega^{j k} \wedge \Omega^{l}{ }_{n} \wedge \theta^{n} \wedge \theta^{*}{ }_{i j k l}-\omega_{i}{ }^{j} \wedge \Omega^{k l} \wedge \theta^{*}{ }_{j k l}\right) .
\end{aligned}
$$

The expression for $\sigma_{i}$ and $\tau_{i}$ are motivated by 
Theorem 2.1. The following conditions a), b), c) are equivalent:

a) $d \tau_{i}=0$

b) $\tau_{i}=d \sigma_{i}$,

c) $\omega^{i}{ }_{j}$ is torsion-free and Ricci-flat.

Proof. Suppose that $d \tau_{i}=0$. By equating to zero in the right-hand side of (2.4) forms of different vertical degree we find that

$$
\left(\omega_{i}{ }^{n} \wedge \omega^{j k} \wedge \theta^{*}{ }_{n j k l}-\omega_{n}^{j} \wedge \omega^{n k} \wedge \theta^{*}{ }_{i j k l}\right) \wedge \Theta^{l}=0
$$

which is equivalent to

$$
\Theta^{i}=0
$$

and

$$
\omega^{j k} \wedge \Omega_{n}^{l} \wedge \theta^{n} \wedge \theta^{*}{ }_{i j k l}-\omega_{i}{ }^{n} \wedge \Omega^{j k} \wedge \theta^{*}{ }_{n j k}=0
$$

which by virtue of $(2.5)$ is equivalent to

$$
\Omega^{j k} \wedge \theta^{*}{ }_{i j k}=0 \text {. }
$$

Because of the well-known identity [8]

$$
-1 / 2 \Omega^{j k} \wedge \theta^{*}{ }_{i j k}=G_{i}^{j} \theta^{*}{ }_{j}, \quad G_{j}^{i}=R^{i}{ }_{j}-R / 2 \delta^{i}{ }_{j},
$$

this implies that $\omega^{i}$ is Ricci-flat. Suppose now that $\omega^{i}{ }_{j}$ is torsion-free and Ricci-flat. Formula (2.3) implies then that $d \sigma_{i}=\tau_{i}$. Condition b) obviously implies condition a).

The vacuum field equations are thus equivalent to the integrability conditions of a $1^{\text {st }}$-order system of differential equations which is in fact the conservation law for the energy-momentum as we shall see below.

This theorem can be generalized to include the case of an arbitrary prescribed Einstein tensor $G_{i j}$ provided that the source term satisfies a conservation equation with respect to the connection $\omega^{i}{ }_{j}$ which makes it compatible with the Bianchi identities. Let $t_{i}$ be an $\mathbb{R}^{n}$-valued horizontal $(n-1)$-form on $O(M, g)$ such that

$$
D t_{i} \equiv d t_{i}+\omega_{i}^{j} \wedge t_{j}=0 \text {. }
$$

Then we have

Theorem 2.2. The following conditions a), b), c) are equivalent:

a) $d\left(\tau_{i}+t_{i}\right)=0$

b) $\tau_{i}+t_{i}=d \sigma_{i}$

c) $\omega^{i}{ }_{j}$ is torsion-free and satisfies the equation,

$$
1 / 2 \Omega^{j k} \wedge \theta^{*}{ }_{i j k}+t_{i}=0 .
$$

Proof. Suppose that $d\left(\tau_{i}+t_{i}\right)=0$. As before we find (2.5) and using the conservation equation (2.8) we find (2.9). Suppose now that $\omega^{i}{ }_{j}$ is torsion-free and satisfies Eq.(2.9). Formula (2.3) implies then that $d \sigma_{i}=\tau_{i}+t_{i}$. Again condition b) obviously implies condition a).

If we write $t_{i}=T_{i}{ }^{j} \theta^{*}$, then (2.9) can be written $G_{i j}=T_{i j}$. Therefore when $M$ is the space-time manifold of General Relativity $t_{i}$ can be considered as the energy- 
momentum density of the material sources written as an $(n-1)$-form on the bundle $O(M, g)$. The condition a) of the theorem can be then seen as a conservation law and $\tau_{i}$ interpreted as a density of gravitational energy-momentum on $O(M, g)$; various pseudo-tensors which represent the gravitational energy-momentum density on $M$ can be obtained from $\tau_{i}$ by taking local sections $s$ of $O(M, g)$.

If $M$ is lorentzian and $\Sigma$ a space-like hypersurface in $M$ over which $O(M, g)$ has a section then an integral quantity $P_{i}(\Sigma)$ can be defined by the equation

$$
P_{i}(\Sigma)=\int_{\Sigma} s^{*}\left(\tau_{i}+t_{i}\right)=\int_{\partial \Sigma} d\left(s^{*} \sigma_{i}\right)
$$

If $\Sigma$ is closed, that is, if the boundary $\partial \Sigma$ of $\Sigma$ does not exist, then $P_{i}$ vanishes. If $M$ is asymptotically flat as the radial distance $r$ from some center tends to infinity and if $\partial \Sigma$ is the $(n-2)$-sphere at infinity, then $P_{i}(\Sigma)$ can be considered as the total energymomentum of the system provided that the integral over $\partial \Sigma$ is finite. If the sources are of compact support there are sections $s$ of $O(M, g)$ such that the components $s^{*}\left(\omega_{j}^{i}\right)$ of the connection form tend to zero as $r^{-n+2}$. In this case the right-hand side of (2.10) is finite since $\sigma_{i}$ is linear in $\omega^{i}{ }_{j}$. The expression (2.1) is the only non-trivial $\mathbb{R}^{n}$-valued $(n-2)$-form which is linear in $\omega_{j}^{i}$.

\section{The Generalized Lagrangians}

When the torsion vanishes Eq. (2.9) is a second-order equation for the metric which can be derived from the lagrangian $L=1 / 2 L_{(1)}+L_{m}$, where $L_{(1)}$ is the EinsteinHilbert lagrangian (a basic $n$-form)

$$
L_{(1)}=\Omega^{i j} \wedge \theta^{*}{ }_{i j},
$$

and $L_{m}$ is the matter lagrangian whose variation yields the source term $t_{i}$. If $n \geqq 5$ the Einstein-Hilbert lagrangian in not the most general lagrangian which yields second-order field equations even in one includes a cosmological constant.

For each integer $p$ with $0 \leqq 2 p \leqq n$, define the $n$-form

$$
L_{(p)}=\left(\Omega^{p}\right)^{i_{1} \cdots i_{2 p}} \wedge \theta_{i_{1} \cdots i_{2 p}},
$$

where $\Omega^{p}$ is the $p^{\text {th }}$ power of $\Omega:\left(\Omega^{p}\right)^{i_{1} \cdots i_{2 p}}=\Omega^{i_{1} i_{2}} \wedge \cdots \wedge \Omega^{i_{2 p-1} i_{2 p}}$.

When $n$ is even, $L_{(n / 2)}$ is closed and represents the Euler class in dimension $n$. It is locally exact and so it does not contribute to the field equations. Using the Kroneker $\delta$-symbol and the volume form $\operatorname{vol}_{M}$ on $M_{n}, L_{(p)}$ can be written in the form.

$$
L_{(p)} \propto \delta_{j_{1} \cdots j_{2 p}}^{i_{1} \cdots i_{2 p}} R_{i_{1} i_{2}}^{j_{1} j_{2}} \cdots R_{i_{2 p-1} i_{2 p}}^{j_{2 p-1} j_{2 p}} \operatorname{vol}_{M} .
$$

$L_{(p)}$ can also be expressed without using the $\delta$-symbol. For example the form $L_{(2)}$ can be written as

$$
L_{(2)} \propto\left(R^{i j k l} R_{i j k l}-4 R^{i j} R_{i j}+R^{2}\right) \operatorname{vol}_{M} .
$$

The most general lagrangian which yields $2^{\text {nd }}$-order field equations can be written as a linear combination of the $L_{(p)}$ [9]:

$$
L_{g}=\sum_{1 \leqq p<n / 2} \beta_{p} \kappa^{2(2-p)} L_{(p)} .
$$


The $\beta_{p}$ are real numbers and we shall set the mass scale $\kappa$ equal to 1 . A possible cosmological term $(p=0)$ can be included in the matter lagrangian $L_{m}$.

If we suppose that the torsion vanishes, from this lagrangian we obtain the equations.

$$
\sum \beta_{p}\left(\Omega^{p}\right)^{i_{1} \cdots i_{2 p}} \wedge \theta^{*}{ }_{i i_{1} \cdots i_{2 p}}+t_{i}=0 .
$$

Einstein's equations are obtained by taking $\beta_{1}=1 / 2$ and $\beta_{p}=0$ for $p \geqq 2$.

In Theorem 2.2 we expressed Einstein's equations as a conservation equation using the $(n-2)$-form $\tau_{i}$. The generalized equation (3.6) can also be written as a conservation equation using a generalization of $\tau_{i}$. Let $\omega_{j}{ }_{j}$ be an arbitrary connection on $O(M, g)$ and define for $p \geqq 1$ the $\mathbb{R}^{n}$-valued $(n-2)$-forms $\sigma^{(p)}{ }_{i}$ and $(n-1)$-form $\tau^{(p)}{ }_{i}$ on $O(M, g)$ by the formulae

$$
\begin{aligned}
\sigma_{i}^{(p)} & =-\omega^{j k} \wedge \theta^{*}{ }_{i j k i_{3} \cdots i_{2 p}} \wedge\left(\Omega^{p-1}\right)^{i_{3} \cdots i_{2 p}} \\
\tau^{(p)} & =\left(\omega_{i}{ }^{j} \wedge \omega^{k l} \wedge \theta^{*}{ }_{j k l i_{3} \cdots i_{2 p}}-\omega^{j}{ }_{l} \wedge \omega^{l k} \wedge \theta^{*}{ }_{i j k i_{3} \cdots i_{2 p}}\right) \wedge\left(\Omega^{p-1}\right)^{i_{3} \cdots i_{2 p}} .
\end{aligned}
$$

It is easy to verify that they satisfy the following identities

$$
\begin{aligned}
& d \sigma_{i}^{(p)}=\tau_{i}^{(p)}+\left(\Theta^{j} \wedge \omega^{k l} \wedge \theta^{*}{ }_{i j k l i \cdots i_{2 p}}-\Omega^{j k} \wedge \theta^{*}{ }_{i j k i_{9} \cdots i_{2 p}}\right) \wedge\left(\Omega^{p-1}\right)^{i_{3} \cdots i_{2 p}}, \\
& d \tau^{(p)}{ }_{i}=\Theta^{m} \wedge\left(\omega_{i}^{l} \wedge \omega^{j k} \wedge \theta^{*}{ }_{j k l m i_{3} \cdots i_{2 p}}-\omega^{j}{ }_{n} \wedge \omega^{n k} \wedge \theta^{*}{ }_{i j k m i_{3} \cdots i_{2 p}}\right) \wedge\left(\Omega^{p-1}\right)^{i_{3} \cdots i_{2 p}} \\
& +\left(\omega^{j k} \wedge \Omega_{n}^{l} \wedge \theta^{n} \wedge \theta^{*}{ }_{i j k l i_{3} \cdots i_{2 p}}-\omega_{i}{ }^{j} \wedge \Omega^{k l} \wedge \theta^{*}{ }_{j k l i_{3} \cdots i_{2 p}}\right) \wedge\left(\Omega^{p-1}\right)^{i_{3} \cdots i_{2 p}}
\end{aligned}
$$

Define

$$
\sigma_{i}=\sum \beta_{p} \sigma_{i}^{(p)}, \quad \tau_{i}=\sum \beta_{p} \tau^{(p)}{ }_{i}
$$

Then we have the following generalization of Theorem 2.2:

Theorem 3.1. The following conditions a), b), c) are equivalent:

a) $d\left(\tau_{i}+t_{i}\right)=0$

b) $\tau_{i}+t_{i}=d \sigma_{i}$

c) the torsion and curvature satisfy the equations.

$$
\begin{aligned}
\sum \beta_{p} \Theta^{m} \wedge \theta^{*}{ }_{j k l m i_{3} \cdots i_{2 p}} & \wedge\left(\Omega^{p-1}\right)^{i_{3} \cdots i_{2 p}}=0, \\
\sum \beta_{p}\left(\Omega^{p}\right)^{i_{1} \cdots i_{2 p}} & \wedge \theta^{*}{ }_{i i_{1} \cdots i_{2 p}}+t_{i}=0 .
\end{aligned}
$$

Proof. The proof is the same as that of Theorem 2.2 if one uses (3.9), (3.10) instead of (2.3), (2.4).

Theorem 2.2 is particular case of this theorem with $\beta_{1}=1 / 2$ and $\beta_{p}=0$ for $p \geqq 2$. Equation (3.11) does not imply that the torsion vanishes except in this case. If the torsion does vanish, Eq. (3.6) is obviously equivalent to (3.11). Any solution to Eqs. (3.6) yields therefore by the theorem a conservation equation, and an integral quantity $P_{i}(\Sigma)$ can be defined as before (Eq. (2.10)). In the asymptotically flat case only the $p=1$ term contributes to the integral over $\partial \Sigma$ at infinity. 


\section{The 4-dimension Case}

In this section the signature is taken to be the Lorentzian signature $(-,+,+,+)$. Greek indices take the values $0,1,2,3$ and Latin indices the values 1,2,3. As before all indices are form indices. Introduce the self-dual and anti-self-dual parts of the connection

$$
\begin{aligned}
& \omega_{\lambda \mu}^{(+)}=1 / 2\left(\omega_{\lambda \mu}-i / 2 \varepsilon_{\lambda \mu \rho \sigma} \omega^{\rho \sigma}\right), \\
& \omega^{(-)}{ }_{\lambda \mu}=1 / 2\left(\omega_{\lambda \mu}+i / 2 \varepsilon_{\lambda \mu \rho \sigma} \omega^{\rho \sigma}\right) .
\end{aligned}
$$

These are complex quantities and one is the complex conjugate of the other. The 2-form $\sigma_{\lambda}$ and the 3-form $\tau_{\lambda}$ can be written in terms of $\omega_{\lambda \mu}^{( \pm)}$:

$$
\begin{aligned}
\sigma_{\lambda} & =-i\left(\omega^{(+)}{ }_{\lambda \mu}-\omega^{(-)}{ }_{\lambda \mu}\right) \wedge \theta^{\mu}, \\
\tau_{\lambda} & =-2 i \omega^{(+)}{ }_{\lambda \mu} \wedge \omega^{(-) \mu}{ }_{\nu} \wedge \theta^{\nu} .
\end{aligned}
$$

With an euclidean signature the corresponding forms $\omega^{( \pm)}$are independent. The above formulae can be conveniently written using quaternion-valued forms. If we denote $i_{k}$ the 3 imaginary quaternionic units and define

$$
\theta=\theta^{0}+\theta^{k} i_{k}, \quad \omega^{( \pm)}=-1 / 2\left( \pm \omega^{0 k}+1 / 2 \varepsilon_{k l m} \omega^{l m}\right) i_{k},
$$

the structure equations become

$$
d \theta+\omega^{(+)} \wedge \theta+\theta \wedge \omega^{(-)}=\Theta, \quad d \omega^{( \pm)}+\omega^{( \pm)} \wedge \omega^{( \pm)}=\Omega^{( \pm)} .
$$

and formulae (4.1), (4.2) become

$$
\begin{aligned}
& \sigma=\omega^{(+)} \wedge \theta-\theta \wedge \omega^{(-)}, \\
& \tau=2 \omega^{(+)} \wedge \theta \wedge \omega^{(-)} .
\end{aligned}
$$

The expression used by Ashtekar and Horowitz [6] in their discussion of the positive-energy theorems is derived from a Minkowski version of (4.4).

Let $\Sigma$ be a space-like hypersurface with a globally defined basis of 1 -forms $\theta^{1}, \theta^{2}, \theta^{3}$. Choose a time coordinate such that $\theta^{0}=d t$ completes this basis to define a section of $O(M, g)$ over $\Sigma$. Define the $3 \times 3$ matrix $a$ by

$$
a_{j}^{i} \theta^{j}=\omega_{\mid \Sigma}^{(+) 0 i}
$$

If we define the local gravitational energy density $\varepsilon$ on $\Sigma$ by the formula

$$
\tau^{0}=\varepsilon \theta^{1} \wedge \theta^{2} \wedge \theta^{3}
$$

we find that it is given in terms of the matrix $a$ by the formula

$$
\varepsilon / 2=\sum a^{*}{ }_{i j} a_{j i}-\left|\sum a_{i i}\right|^{2}=\operatorname{Tr}\left(a^{* T} a\right)-|\operatorname{Tr}(a)|^{2} .
$$

If we decompose the matrix $a$ into its irreducible components, the symmetric tracefree part $S$, the anti-symmetric part $A$ and the trace,

$$
a_{i j}=S_{i j}+A_{i j}+1 / 3 \operatorname{tr}(a) \delta_{i j}
$$

this can be written

$$
\varepsilon / 2=\operatorname{Tr}\left(S^{*} S\right)-\operatorname{Tr}\left(A^{*} A\right)-2 / 3|\operatorname{Tr}(a)|^{2}
$$


Because of our choice of frame the structure equations imply that $A$ is pure imaginary. The total energy is given in the preferred frame by

$$
p^{0}=\int_{\Sigma}\left(G_{0}^{0}+\varepsilon\right) \theta^{1} \wedge \theta^{2} \wedge \theta^{3}=\int_{\partial \Sigma} \sigma^{0} .
$$

Suppose that $M$ is asymptotically flat. Since

$$
\sigma^{0}=2 i A_{i j} \theta^{i} \wedge \theta^{j}
$$

and therefore $\left\|\sigma^{0}\right\|$ is proportional to $\operatorname{Tr}\left(A^{*} A\right)$, it is obvious that there is no appropriate choice of frame in which $A$ can be chosen to vanish.

If $u_{\lambda}$ is any time-like vector field which tends to the unit normal to $\Sigma$ at infinity, $P^{0}$ can also be expressed in the form

$$
P^{0}=-\int_{\partial \Sigma} \sigma^{\lambda} u_{\lambda}=-\int_{\Sigma}\left(\tau_{\lambda} u^{\lambda}+t_{\lambda} u^{\lambda}-\sigma^{\lambda} \wedge d u_{\lambda}\right)
$$

Although the expression for (4.8) is not positive definite, using the constraint conditions $G^{0}{ }_{0} \geqq 0$, the integral quantity $P^{0}$ can be shown to be non-negative. One can prove this by constructing some vector field $u_{\lambda}$ such that the integrand on the right of Eq. (4.11) is never positive. The integrand is not then in general equal to the energy density. Because the spinor connection is constructed directly from $\omega^{\lambda}{ }_{\mu}$ by contracting with Dirac $\gamma$-matrices, the most convenient choice of $u_{\lambda}$ is the current of a spinor field $[3,4]$.

The fact that $P^{0}$ is positive is true in general $n \geqq 4$ dimensions [5] if one uses the form $\tau_{i}$ constructed in Sect. 2. We do not expect a similar result to be valid for the equivalent quantity which is relevant when considering the generalized lagrangians since the constraint conditions on $G^{0}{ }_{0}$ are no longer necessarily satisfied.

\section{Yang-Mills Currents and the Kaluza-Klein Approach}

Let $P=P(M, G)$ be a $G$-principal bundle over the pseudo-Riemannian manifold $M$ of dimension $n$. Let $A$ be a connection form on $P$ with curvature 2-form $F=d A+$ $1 / 2[A, A]$ and let $F^{*}$ be the dual of $F$. Assume that the Lie algebra $\operatorname{Lie}(G)$ of $G$ is semi-simple. Then Theorems 2.1 and 2.2 have the following analogues.

Theorem 5.1. The following conditions a), b) are equivalent.

a) $d\left(-\left[A, F^{*}\right]\right)=0$,

b) $-\left[A, F^{*}\right]=d F^{*}$, that is, the Yang-Mills equations $D F^{*} \equiv d F^{*}+\left[A, F^{*}\right]=0$ are satisfied.

Proof. Suppose b) is true. Then a) follows without any assumption on $G$ Suppose a) is true. From the identity

$$
d\left(-\left[A, F^{*}\right]\right)=-\left[d A, F^{*}\right]+\left[A, d F^{*}\right]=\left[A, D F^{*}\right],
$$

we see that $D F^{*}=0$ since $D F^{*}$ is horizontal and the center of $\operatorname{Lie}(G)$ reduces to 0 .

This theorem can be generalized to include the case of an arbitrary prescribed source term provided the latter satisfies a conservation equation which makes it compatible with the Bianchi identities. Let $j$ be a $\operatorname{Lie}(G)$-valued horizontal $(n-1)$-form on $P$ 
such that

$$
D j \equiv d j+[A, j]=0 .
$$

Then we have

Theorem 5.2. The following conditions a), b) are equivalent

a) $d\left(-\left[A, F^{*}\right]+j\right)=0$,

b) $-\left[A, F^{*}\right]+j=d F^{*}$, that is, the equations

$D F^{*}=j$ are satisfied.

Proof. Suppose b) is true. Then a) follows again without any assumption on $\operatorname{Lie}(G)$. Suppose a) is true. Using the conservation equation we have

$$
d\left(-\left[A, F^{*}\right]+j\right)=-\left[d A, F^{*}\right]+\left[A, d F^{*}\right]-[A, j]=\left[A, D F^{*}-j\right]
$$

and we see now that $D F^{*}=j$.

Remark. If one drops the assumption on $\operatorname{Lie}(G)$ then condition b) must be replaced by condition

$\left.\mathrm{b}^{\prime}\right) D F^{*}-j$ takes its values in the center of Lie $(G)$.

Clearly the forms $F^{*}$ and $-\left[A, F^{*}\right]$ are the analogues of the forms $\sigma_{i}$ and $\tau_{i}$ which we introduced in Sect. 2 just as $j$ is the analogue of $t_{i}$. So we can interpret $-\left[A, F^{*}\right]$ as the charge density on $P$ of the Yang-Mills potential $A$.

It is worth noticing also that there is a strong connection between the Theorems of Sect. 2 and this section and the fact that the Einstein equations and the YangMills equations are the integrability conditions of the corresponding linear system obtained by linearizing about a generic field configuration. For more details see $[10,11]$.

The analogy between the above theorems and those of Sect. 2 can be best understood in the context of a Kaluza-Klein unification of gravity and Yang-Mills fields. Denote by $M^{\prime}$ the $G$-principal bundle $P$ over $M$ and by $p$ the projection onto $M$. On $M^{\prime}$ consider the metric $g^{\prime}$ given by $g^{\prime}=p^{*}(g)+\mu^{-2} k(A, A)$, where $k$ is the Cartan-Killing bilinear form on $\operatorname{Lie}(G)$. The constant $\mu$ has the dimensions of mass. We have chosen units such that $8 \pi G=1$, where $G$ is Newton's constant. So $2 \mu^{2}$ is equal to the square of the Yang-Mills charge. Let $A^{a}, 1 \leqq a \leqq \operatorname{dim}(G)$, be the components of the connection form on $P$ in a fixed orthonormal basis $e_{a}$ of $\operatorname{Lie}(G)$. For any $x^{\prime}$ in $M^{\prime}$ and any orthonormal basis at $p\left(x^{\prime}\right)$ with dual basis $\theta^{\alpha}, 1 \leqq \alpha \leqq n$, a unique orthonormal basis can be constructed at $x^{\prime}$ with dual basis $\theta^{i}, i=(\alpha, a)$, given by the formulae

$$
\theta^{\prime \alpha}=p^{*}\left(\theta^{\alpha}\right), \quad \theta^{\prime a}=\mu^{-1} A^{a} .
$$

The $\theta^{\prime i}$ are the components of the canonical form on a subbundle $O^{\prime}$ of $O\left(M^{\prime}, g^{\prime}\right)$.

If $\omega^{\alpha}{ }_{\beta}$ is a connection form on $M$ and if $C^{a}{ }_{b c}$ are the structure constants of $G$ then a connection form $\omega^{i}{ }_{j}$ on $O^{\prime}$ is given by the equations [12]

$$
\begin{aligned}
\omega^{\prime a}{ }_{b} & =-\mu / 2 C_{b c}^{a} \theta^{\prime c}, \\
\omega^{\prime a}{ }_{\alpha} & =1 / 2 \mu F^{a}{ }_{\alpha \beta} \theta^{\prime \beta}, \\
\omega^{\prime \alpha}{ }_{\beta} & =p^{*}\left(\omega^{\alpha}{ }_{\beta}\right)+1 / 2 \mu F^{\alpha}{ }_{a \beta} \theta^{\prime a}, \quad F_{\alpha a \beta}=-F_{a \alpha \beta} .
\end{aligned}
$$


The functions $F_{a \alpha \beta}$ are the coefficients of the field strength $F$ in the frame $\theta^{\alpha}$ :

$$
F=F^{a} e_{a}=1 / 2 F_{\alpha \beta}^{a} \theta^{\alpha} \wedge \theta^{\beta} e_{a} .
$$

If $\omega^{\alpha}{ }_{\beta}$ is torsion free then so is $\omega^{\prime i}$.

The forms $\sigma_{i}, \tau_{i}$ and $t_{i}$ which we introduced in Sects. 2 and 3 have analogues $\sigma_{i}^{\prime}, \tau_{i}^{\prime}$ and $t_{i}^{\prime}$ on $O^{\prime}$. The latter can be expanded into horizontal and vertical components. The horizontal components are related to the forms $\sigma_{i}, \tau_{i}$ and $t_{i}$ on $O(M, g)$ and the vertical components to the corresponding Yang-Mills quantities. Let $\Sigma$ be a local $(n-1)$-hypersurface in $M$ and $\Sigma^{\prime}=p^{*}(\Sigma)$ the corresponding hypersurface in $M^{\prime}$. A straightforward calculation yields the following relation between $\sigma_{i}^{\prime}$ on the one hand and $\sigma_{\alpha}$ and $F^{*}{ }_{a}$ on the other:

$$
\begin{aligned}
\sigma_{\alpha \mid \Sigma^{\prime}}^{\prime} & =\sigma_{\alpha} \wedge \operatorname{vol}_{G}, \\
\sigma_{a \mid \Sigma^{\prime}}^{\prime} & =1 / 2 \mu F^{*}{ }_{a} \wedge \operatorname{vol}_{G} .
\end{aligned}
$$

Define the Yang-Mills energy-momentum density $T^{Y M}{ }_{\alpha \beta}$ as

$$
T^{Y M}{ }_{\alpha \beta}=-1 / 2 \mu^{2}\left(F_{a \alpha \gamma} F^{a}{ }^{\gamma}-1 / 4 g_{\alpha \beta} F_{a \gamma \delta} F^{a \gamma \delta}\right) \text {. }
$$

Then a similar calculation yields

$$
\begin{aligned}
& \tau_{\alpha \mid \Sigma^{\prime}}^{\prime}=1 / 8 C_{a b c} C^{a b c} \theta_{\alpha}^{*} \wedge \operatorname{vol}_{G}+\left(\tau_{\alpha}+T^{Y M}{ }_{\alpha}{ }^{\beta} \theta_{\beta}{ }_{\beta}\right) \wedge \operatorname{vol}_{G}, \\
& \tau_{a \mid \Sigma^{\prime}}^{\prime}=1 / 2 C_{a b c} F^{* b} \wedge \theta^{c} \wedge \operatorname{vol}_{G}=-1 / 2 \mu\left[A, F^{*}\right]_{a} \wedge \operatorname{vol}_{G} .
\end{aligned}
$$

If we decompose in a similar way the external-source form $t_{i}$

$$
\begin{aligned}
& t_{\alpha \mid \Sigma^{\prime}}^{\prime}=t_{\alpha} \wedge \operatorname{vol}_{G}, \\
& t_{a \mid \Sigma^{\prime}}^{\prime}=1 / 2 \mu j_{a} \wedge \operatorname{vol}_{G},
\end{aligned}
$$

then condition a) of Theorem 2.2 yields the following equations:

$$
\begin{aligned}
d\left(\tau_{\alpha}+t_{\alpha}+T_{\alpha}^{Y M}{ }_{\alpha} \theta^{*}{ }_{\beta}+1 / 8 C_{a b c} C^{a b c} \theta_{\alpha}\right) & =0, \\
d\left(-\left[A, F^{*}\right]_{a}+j_{a}\right) & =0 .
\end{aligned}
$$

The first of these equations is the same as the original condition a) but on $O(M, g)$ and with an effective external source given by

$$
t^{\mathrm{eff}}=t_{\alpha}+T^{Y M}{ }_{\alpha}^{\beta} \theta_{\beta}{ }_{\beta}+1 / 8 C_{a b c} C^{a b c} \theta_{\alpha}^{*} .
$$

There are two additional terms, the Yang-Mills energy-momentum density and a cosmological constant. Equation (5.11) is the same as condition a) of Theorem 5.2.

Acknowledgements. The authors would like to thank Jean Ginibre and David Robinson for enlightening conversations.

\section{References}

1. Deser, S., Teitelboim, C.: Supergravity has positive energy. Phys. Rev. Lett. 39, 249 (1977)

2. Schoen, R., Yau, S.-T.: On the proof of the positive mass conjecture in general relativity. Commun. Math. Phys., 6, 45 (1979); The energy and the linear momentum of space-times in general relativity. 79, 47 (1981). The proof of the positive mass theorem. II, 79, 231 (1981) 
3. Witten, E.: A new proof of the positive energy theorem. Commun. Math. Phys. 80, 381 (1981)

4. Nester, J. M.: A new gravitational energy expression with a simple positivity proof. Phys. Lett. 83A, $241(1981)$

5. Taub, A. H.: Positivity of energy in $n$-dimensional Lorentzian spaces. Lett. Math. Phys. 10, 55 (1985)

6. See, for example, Ashtekar, A.: On the Hamiltonian of general relativity. VII ${ }^{\text {th }}$ International Congress on Mathematical Physics. Boulder, Colorado 1983

7. Sparling, G.A.J.: Twistors, spinors and the Einstein vacuum equations. University of Pittsburg preprint (1984)

8. Thirring, W.: Classical field theory. Berlin, Heidelberg, New York: Springer 1979

9. Lovelock, D.: The Einstein tensor and its generalizations. J. Math. Phys. 12, 498 (1971); Muto, Y.: Tensor 29, 125 (1975); Paterson, E. M.: J. Lond Math. Soc. 2, 349 (1981); Müller-Hoissen, F.: Spontaneous compactification with quadratic and cubic curvature terms. Phys. Lett. 163B, 106 (1985); Deruelle, N., Madore, J.: On the vanishing of the cosmological constant. Phys. Lett. 114A, 185 (1986)

10. Dubois-Violette, M.: Einstein equations. Yang-Mills equations and classical field theory as compatibility conditions of linear partial differential operators. Phys. Lett. 119B, 157 (1982); Remarks on the local structure of Yang-Mills and Einstein equations. 131B, 323 (1983); Geom. Phys. 1, 139 (1984)

11. Gasqui, J.: Compositio Mathematica 47, 43 (1982)

12. See, for example, Jensen, G. R.: J. Differ Geom. 8, 599 (1973)

Communicated by S.-T. Yau

Received February 18, 1986 
